\title{
Brustkrebs: Kognitive Leistungsfähigkeit und proinflammatorische Zytokine
}

\begin{abstract}
Frauen mit Brustkrebs können bereits vor Beginn der adjuvanten Therapie eine neurokognitive Dysfunktion aufweisen. Über die Pathomechanismen weiß man wenig - möglicherweise beeinträchtigen schon vor der Operation erhöhte proinflammatorische Zytokine die kognitive Leistungsfähigkeit.
\end{abstract}

\begin{abstract}
nsgesamt 174 postmenopausale Frauen mit neu diagnostiziertem Brustkrebs wurden vor der Operation umfassend neuropsychologisch untersucht (kognitive Funktion, Stimmung und Fatigue). Gleichzeitig wurden bei ihnen sowie bei 88 altersgematchten Frauen ohne Brustkrebs 3 zirkulierende Zytokine bestimmt: Interleukin-6 (IL-6), Interleukin-1-Rezeptorantagonist (IL-1-RA) und löslicher TNF-Rezeptor Typ 2 (sTNFR2). Mithilfe multivariater Regressionsanalysen wurde die Rolle der Zytokine für die neurokognitive Leistungsfähigkeit betrachtet.

Das verbale Gedächtnis war die einzige kognitive Domäne, die sich nach Kontrolle der Kovariaten statistisch signifikant zwischen der Patientinnen-
\end{abstract}

und der Kontrollgruppe unterschied $(p=0,02)$. Von den 3 gemessenen $\mathrm{Zy}$ tokinen war nur IL-1-RA bei den Krebspatientinnen statistisch signifikant gegenüber der Kontrollgruppe erhöht $(375 \pm 239$ pg/ml vs. $291 \pm 169$ pg/ $\mathrm{ml} ; \mathrm{p}=0,007)$; es erwies sich jedoch nicht als unabhängiger Parameter.

Nach Ausschluss möglicher anderer vorbestehender Einflussfaktoren konnten $6 \%$ der Gesamtvarianz der (verbalen) Gedächtnisleistung auf die Zytokine zurückgeführt werden ( $p=0,01)$. Als Einzelfaktor war speziell ein höherer Spiegel des sTNFR2 mit einer schlechteren Gedächtnisleistung assoziiert, allerdings nur bei den Patientinnen, nicht in der Kontrollgruppe. Die TNF-Werte lagen
Schon bei Brustkrebsdiagnose kann die kognitive Funktion beeinträchtigt sein.

bei den Brustkrebspatientinnen aber nicht höher als in der Kontrollgruppe.

Fazit: Eine Assoziation von TNF und Gedächtnis, wie sie zuvor für Patienten nach Chemotherapie beschrieben wurde, kann bereits vor Beginn jeglicher Therapie inklusive Operation festgestellt werden. Diese Zusammenhänge müssen aber mit größeren Patientenzahlen näher erforscht werden. Brigitte Schalhorn

Patel SK et al. Inflammatory biomarkers, comorbidity, and neurocognition in women with newly diagnosed breast cancer. J Natl Cancer Inst. 2015;107(8). pii: djv131.

\section{Mammakarzinom mit PALB2-Mutation hat schlechtere Prognose}

\author{
Mutationen im PALB2-Gen bringen \\ ein erhöhtes Brustkrebsrisiko mit \\ sich. Unklar war jedoch bisher, \\ welche Prognose Frauen mit PALB2- \\ Mutation haben.
}

Z wischen 1996 und 2012 wurden an 18 Kliniken in Polen Patientinnen mit invasivem Brustkrebs prospektiv erfasst und auf zwei deletäre Mutationen im PALB2-Gen (509_510delGA und 172_175delTTGT) untersucht. Zum Vergleich wurde eine Kontrollgruppe von 4.702 Frauen ohne Karzinom ebenfalls auf diese beiden Mutationen hin gescreent. Beide Kohorten wurden bis Juli 2014 beobachtet, die mediane Followup-Dauer betrug 53,5 Monate.

17.900 Frauen mit Brustkrebs nahmen an der Studie teil, bei 12.529 wurde der Genotyp erfolgreich dargestellt. Eine PALB2-Mutation lag bei 0,93\% der Pati- entinnen und bei $0,21 \%$ der gesunden Kontrollprobandinnen vor. Die Odds Ratio (OR) für ein Mammakarzinom betrug für die Mutationsträgerinnen 4,39 (95\%-Konfidenzintervall [95\%-KI] 2,30-8,37; $\mathrm{p}<0,0001)$. Die 10-JahresGesamtüberlebensrate war bei Frauen mit Brustkrebs und PALB2-Mutation mit 48,0\% (95\%-KI 36,5-63,2) deutlich niedriger als bei Patientinnen mit Brustkrebs ohne diese Mutation $(74,7 \%$, 95\%-KI 73,5-75,8; Hazard Ratio 2,27, 95\%-KI 1,64-3,15; p < 0,0001). Die PALB2-Mutation ist in der Allgemeinbevölkerung selten.

Interessant ist der Vergleich mit der BRCA1-Mutation, die ebenfalls erfasst und bei $0,47 \%$ der Kontrollprobandinnen und bei 3,47\% der Brustkrebspatientinnen festgestellt wurde (OR 7,65). Keine Patientin hatte eine Mutation in beiden Genen. Die 10-Jahres-Gesamtüberlebensrate war bei BRCA1- günsti- ger als bei PALB2-Mutation (72,0 vs. $48,0 \%$ ) und $74,7 \%$ für Frauen ohne eine der beiden Mutationen $(p<0,0001)$. Frauen mit PALB2-Mutation hatten häufiger ein tripelnegatives Mammakarzinom. Als stärkster Einflussfaktor auf das Überleben erwies sich die Tumorgröße zum Zeitpunkt der Diagnose.

Fazit: Frauen mit PALB2-Mutation haben ein erhöhtes Risiko, an Brustkrebs zu erkranken, und zeigen nach Manifestation des Tumors ein vermindertes 10-Jahres-Überleben im Vergleich zu Frauen ohne diese Mutation. Noch nicht an Brustkrebs erkrankten Frauen mit PALB2-Mutation sollte eine verstärkte Vorsorge angeboten werden.

Brigitte Schalhorn

Cybulski C et al. Clinical outcomes in women with breast cancer and a PALB2 mutation: a prospective cohort analysis. Lancet Oncol. 2015; 16(6):638-44. 